\title{
The Silicon:Colloidal Quantum Dot Heterojunction
}

\author{
Silvia Masala, Valerio Adinolfi, Jon-Paul Sun, Silvano Del Gobbo, \\ Oleksandr Voznyy, Illan J. Kramer, Ian G. Hill, \\ and Edward H. Sargent
}

Version Post-Print/Accepted Manuscript

Citation Masala, S., Adinolfi, V., Sun, J., Gobbo, S., Voznyy, O., \& Kramer, I. et (published version) al. (2015). The Silicon:Colloidal Quantum Dot Heterojunction. Advanced Materials, 27(45), 7445-7450. http://dx.doi.org/10.1002/adma.201503212

Publisher's Statement This is the peer reviewed version of the following article: Masala, S., Adinolfi, V., Sun, J., Gobbo, S., Voznyy, O., \& Kramer, I. et al. (2015). The Silicon:Colloidal Quantum Dot Heterojunction. Advanced Materials, 27(45), 7445-7450, which has been published in final form at http://dx.doi.org/10.1002/adma.201503212. This article may be used for non-commercial purposes in accordance with Wiley Terms and Conditions for Self-Archiving.

\section{How to cite TSpace items}

Always cite the published version, so the author(s) will receive recognition through services that track citation counts, e.g. Scopus. If you need to cite the page number of the TSpace version (original manuscript or accepted manuscript) because you cannot access the published version, then cite the TSpace version in addition to the published version using the permanent URI (handle) found on the record page. 


\section{The Silicon:Colloidal Quantum Dot Heterojunction}

Silvia Masala ${ }^{1}$, Valerio Adinolfi ${ }^{2}$, Jon-Paul Sun ${ }^{3}$, Silvano Del Gobbo ${ }^{1}$, Oleksandr Voznyy ${ }^{2}$, Illan J. Kramer², Ian G. Hill ${ }^{3}$, Edward H. Sargent ${ }^{2 *}$

${ }^{1}$ Division of Physical Sciences and Engineering, Solar and Photovoltaics Engineering Center, King Abdullah University of Science and Technology (KAUST), Thuwal 23955-6900, Saudi Arabia

${ }^{2}$ Department of Electrical and Computer Engineering, University of Toronto, 10 King's College Road, Toronto, Ontario M5S 3G4, Canada.

${ }^{3}$ Department of Physics and Atmospheric Science, Dalhousie University, 1459 Oxford Street, Halifax, Nova Scotia, B3H 4R2, Canada.

*Corresponding Author: ted.sargent@utoronto.ca

Crystalline silicon (c-Si) technology dominates electronics and optoelectronics: ultra-large scale of integration of integrated circuits, fast optical devices and efficient solar panels are all established applications based on c-Si. Despite its many virtues, silicon is limited in sensing, communication and light harvesting applications by its electronic bandgap, which does not absorb light beyond $1.1 \mu \mathrm{m}$. The sensitization of c-Si with infrared-sensitive materials is therefore an area of great interest. Colloidal quantum dots offer an infrared bandgap; however, the c-Si:CQD rectifying junction has not yet been developed. Here we 
construct such a junction, but find initially that an energy band mismatch and highly defective c-Si:CQD interface prevent efficient extraction of photocurrent. We then devise a means of functionalizing the c-Si:CQD interface that simultaneously passivates the silicon and CQD terminations, and, further, introduces an interface dipole that produces a favorable band alignment between the two solids. Only by so doing are we then able to demonstrate, for the first time, efficient injection of infrared-generated $(>1.1 \mu \mathrm{m}$ absorbed photon wavelength) photocharges from the CQD layer into silicon. Our integrated c-Si:CQD photodiode achieves a high responsivity - of $0.4 \mathrm{~A} / \mathrm{W}$ - for $1.23 \mu \mathrm{m}$ incident radiation. This materials platform, in contrast to established epitaxial approaches (Ge, III-V compounds), allows for room temperature large-area low cost of fabrication.

Crystalline silicon (c-Si) is widely used in photonic devices as a light-guiding medium because of its high index, low loss, and robust electronic performance. Its utility in light detection ${ }^{1,2}-$ especially in the shorter visible wavelengths of the optical spectrum - makes it the workhorse of digital imaging today. ${ }^{3,4}$ Unfortunately, silicon's $1.1 \mathrm{eV}$ bandgap prevents it from providing photon detection in the short-wavelength infrared (SWIR), i.e. at wavelengths greater than $1.1 \mu \mathrm{m}$. This regime is particularly useful for night-time imaging based on atmospheric nightglow $;^{5}$ as well as in gesture recognition, automated inspection and process quality control, ${ }^{6}$ medical imaging, ${ }^{7,8}$ and on-chip communications. ${ }^{9}$ 
There exist numerous prior reports of non-monolithic (hybrid) integration onto silicon of separately epitaxially-grown materials such as III-V compound semiconductors. This packagelevel integration increases cost and device size and often demands bonding processes incompatible with complementary metal oxide semiconductor (CMOS) technology. ${ }^{10}$ Further, the materials comprising III-V compounds can contaminate silicon ${ }^{11}$, strongly affecting its electronic properties; these problems limit the potential for a facile and direct integration of III-V compounds onto silicon. Crystalline germanium has been extensively used to create heterojunction devices with cSi, but also suffers from complexity of fabrication and a reliance on high-temperature growth. ${ }^{12}$

Graphene:c-Si heterostructures have extended the range of light sensitivity of silicon devices to the mid-infrared, but only when combined in a waveguide structure. ${ }^{13}$ Graphene's weak optical absorption - in the vicinity of $2 \%$ per monolayer ${ }^{14,15}$ - requires sophisticated optical structures ${ }^{16}$ to provide acceptable sensitivities when combined with $\mathrm{c}-\mathrm{Si}$ in a diode structure. The requirement of multi-micrometer interaction lengths stands in the way of applications such as imaging that require $\sim 1 \mu \mathrm{m}$ pixel dimensions.

The realization of a high-performance, room-temperature-processed silicon-integrated heterojunction that is responsive beyond $1.1 \mathrm{um}$ remains an unmet challenge as of this report. ${ }^{17,18}$

Colloidal quantum dots (CQDs) have seen rapid growth in the last decade in optoelectronics because of their room-temperature solution processing, low cost and spectral tunability. ${ }^{19}$ This class of semiconductor solids has shown considerable promise in photovoltaics ${ }^{20,21}$ and photodetection, including at SWIR wavelengths. ${ }^{22,23}$ Development of a heterojunction device 
between a CQD solid and electronic-grade silicon would enable a monolithically-integrated, tunable and SWIR-responsive device platform.

The combination of CQDs and silicon has primarily been used to enhance absorption of structured p-n silicon solar cells in the visible range. ${ }^{24} \mathrm{c}-\mathrm{Si}$ :CQD rectifying solar cells have been presented, but to date exhibit no extraction of carriers generated using sub-silicon-bandgap (> $1.1 \mu \mathrm{m})$ illumination. ${ }^{25,26}$ The reasons for this failure of the c-Si:CQD heterointerface have, to this point, been unexplored and unexplained.

Here we investigate the c-Si:CQD monolithically-integrated rectifying junction. We find that a specific and novel combination of process conditions is required to achieve the efficient collection of SWIR photogenerated charges. We then proceed to build an efficient photodetector using a room-temperature solution-processed material compatible with CMOS technology. 
a)

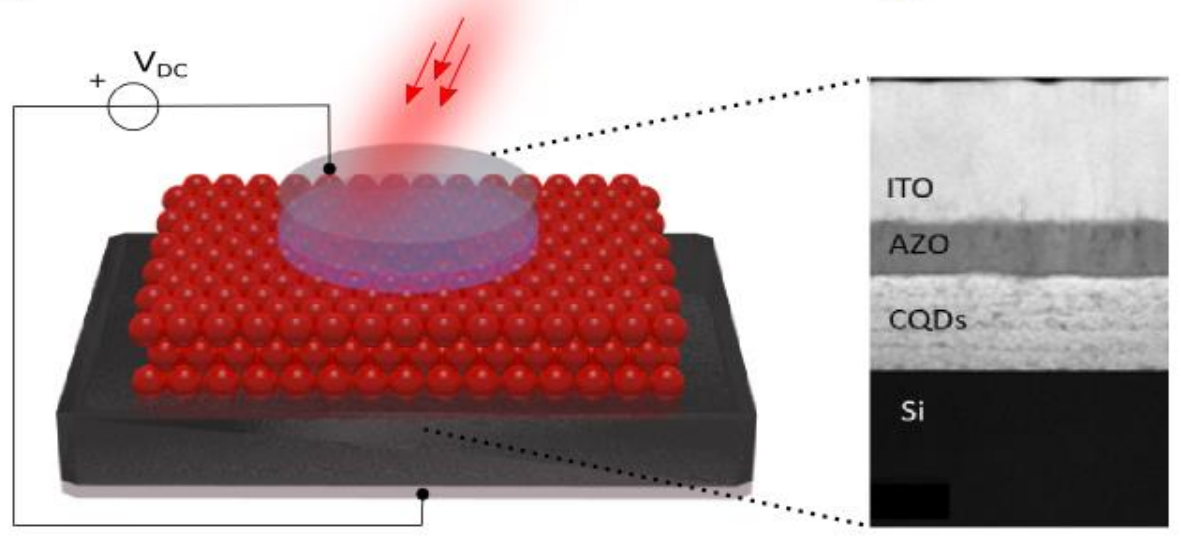

c)

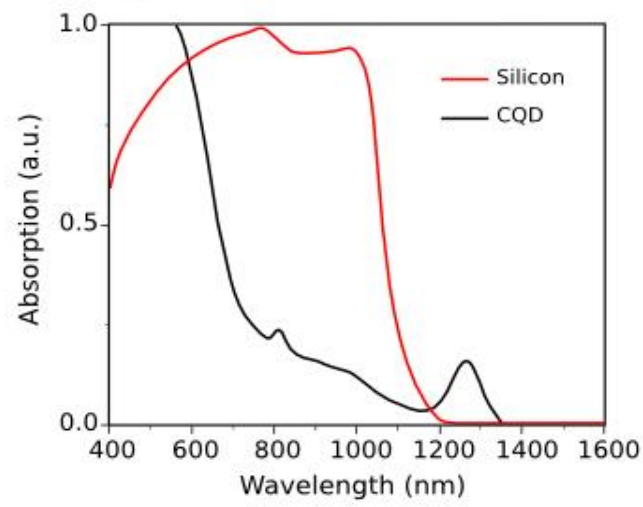

b)

d)

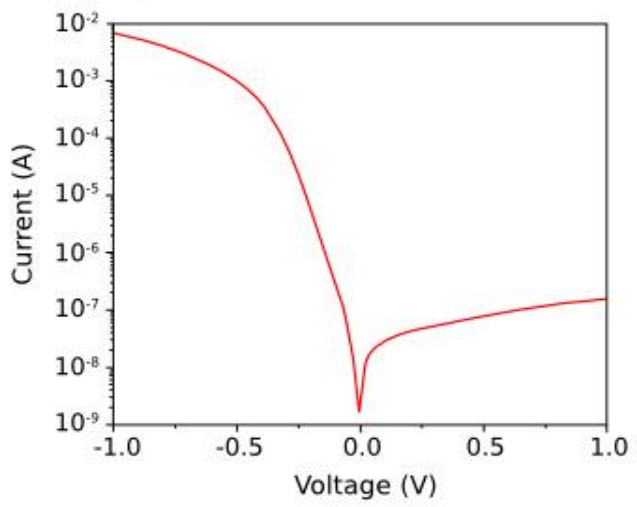

Figure 1. c-Si:CQD junction and materials properties. a) Schematic of the c-Si:CQD photodiode architecture: p-type silicon is coated with the CQD layer; the transparent (illuminated) top contact is realized with aluminum-doped zinc oxide (AZO) and indium tin oxide (ITO); and the silicon side of the junction is contacted using aluminum on the back side. b) STEM cross-section of the diode. c) Measured absorption of crystalline silicon and CQDs. The CQDs are characterized by an excitonic peak centered at 1230 nm. d) c-Si:CQDs current-voltage plot showing rectification.

The diode investigated here (Figure 1a-b) is based on a p-type silicon wafer $(<100\rangle$ crystalline orientation) that we overcoat using a $150 \mathrm{~nm}$ film of halide-treated n-type PbS CQD (1 eV bandgap). The diode is top illuminated through a transparent metal oxide layer deposited on the 
CQD film. In order to collect photocharges efficiently from the CQD layer, the following three requirements must be fulfilled simultaneously:

(i) The c-Si and CQDs must be in intimate contact while employing an effective passivation of interfacial defects;

(ii) The band alignment of the two semiconductors must not result in an energetic barrier for the photo-carriers generated in the CQD film;

(iii) A depletion region supplying a built-in charge extracting electric field must extend into the CQD layer to overcome limited electric transport in this semiconductor. ${ }^{27}$ Simultaneously satisfying conditions (i), (ii) and (iii) should enable efficient detection of wavelengths of light inaccessible to the c-Si layer, but to which the CQD are sensitive.

We started our study using a common c-Si interface treatment, the application of hydrofluoric acid (HF) on the pre-cleaned silicon surface. This procedure removes the native oxide covering the silicon surface and passivates superficial dangling bonds using hydrogen. ${ }^{28-30}$ Deposition of a CQD film on HF-treated c-Si produced a rectifying junction; however, the forward-to-reverse current ratio was undesirably low and the onset of a breakdown is seen at a small voltage of $\sim 6 \mathrm{~V}$ (see S.I. Figure 1). We interpreted this result as evidence that condition (i) was not fully satisfied: intimate contact between silicon and CQD had been realized, but with poor passivation of the interface states, thus limiting the device performance.

Halides have been shown to be superior to hydrogen in passivating silicon surfaces $;{ }^{31-33}$ further, halide treatments of CQD solids were recently found to result in well passivated, n-type films. ${ }^{34}$, ${ }^{35}$ A halide surface treatment would therefore promote compatibility at the c-Si:CQD interface, 
and also enable a p-type c-Si:n-type CQD rectifying junction. Pursuing this route, we used iodine to modify the silicon surface chemically. Following the treatment, we investigated adsorbed iodine on the bare silicon surface using X-Ray Photoelectron Spectroscopy (XPS) (S.I. Figure 2, 3). The c-Si:CQD diode built using this passivation technique showed improved rectification compared to the HF-treated device (Figure 1d, S.I. Figure 1); its reverse saturation current was fully one order of magnitude lower. An accompanying elimination of low-voltage device breakdown was also observed. Using the transient photoconductance decay technique ${ }^{36}$ (S.I. Table 1) we measured charge carrier lifetime and confirmed that the improved rectification arose from a reduced trap state density in iodine-passivated samples compared to HF acid-passivated samples. The carrier lifetime is inversely proportional to the density of traps and therefore is an indicator of interface passivation 
a)
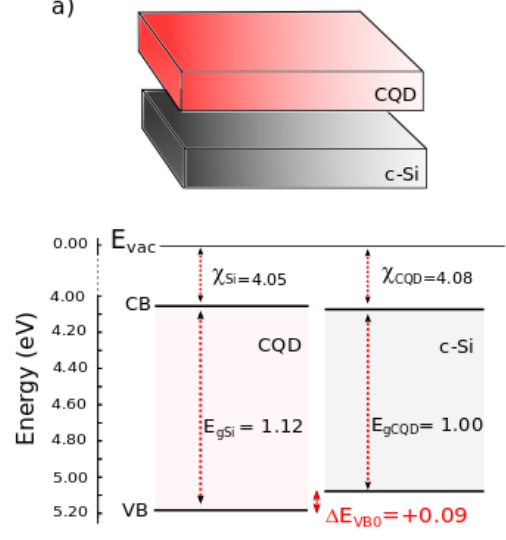

c)

\begin{tabular}{|c|c|c|}
\hline \multicolumn{3}{|c|}{ Measured Values } \\
\hline & $\Delta \mathrm{E}_{\text {Vac }}$ & $\Delta \mathrm{E}_{\mathrm{VB}}$ \\
\hline $\mathrm{I}_{2}$ & -0.23 & +0.32 \\
\hline $\mathrm{Br}_{2}$ & 0.14 & -0.05 \\
\hline $\mathrm{CH}_{3} \mathrm{l}$ & 0.10 & -0.02 \\
\hline
\end{tabular}

b)
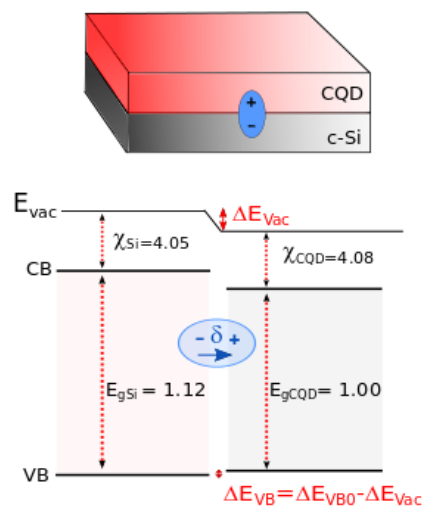

d)

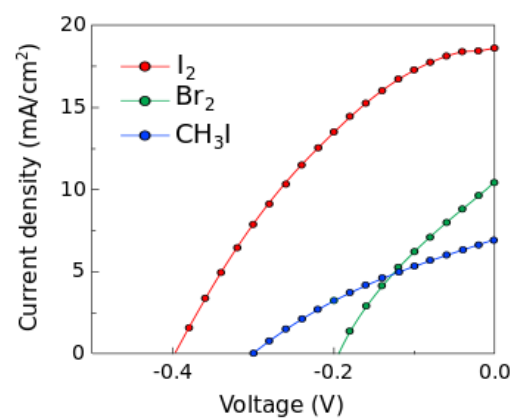

Figure 2. Schematic of the effect of surface dipole on c-Si energy band and current density - voltage characteristics under $100 \mathrm{~mW} \mathrm{~cm}^{-2}$ AM1.5 light. Energy diagram of the c-Si:CQDs diode a) without and b) with the effect of the interface dipole. The dipole introduces a discontinuity $\Delta \mathrm{E}_{\mathrm{vac}}$ in the vacuum level at interface. c) Vacuum level offset $\Delta \mathrm{E}_{\mathrm{vac}}$ and valence band offset $\Delta \mathrm{E}_{\mathrm{vB}}$ as measured from UPS and Kelvin Probe measurements for different halogen treatment of the silicon. d) Experimental J-V curves for each c-Si:CQD device.

While impressive rectification was achieved by satisfying requirement (i), spectral external quantum efficiency (EQE) measurements clearly indicated the lack of photocurrent collected from the CQD layer, i.e. beyond $1.1 \mu \mathrm{m}$ (S.I. Figure 4). We therefore proceeded to analyze the energy band alignment of the heterojunction to see if it was limiting the extraction of photocharges from the CQD layer (requirement (ii)). In Figure 2a the energy diagram of the junction is drawn according to the Anderson rule for the electron affinities. ${ }^{37}$ Published conduction and valence band energies were used for $\mathrm{c}-\mathrm{Si}$ while the energetic picture of the CQD film was determined by cyclic voltammetry and optical absorption measurements (S.I. Figure 5). The c-Si:CQDs band lineup 
resulting from this model produces a type-I heterojunction (Figure 2a) with an undesired barrier of $0.09 \mathrm{eV}$ in the valence band. This blocks the flow of photoholes from the CQDs to the silicon. This barrier prevents the new diode from sensing radiation beyond $1.1 \mu \mathrm{m}$.

By adding a dipole to the interface between the c-Si surface and the CQD film, we can realize the efficient collection of photocharges across the c-Si:CQD boundary. ${ }^{38}$ Chemisorbed molecular species can form surface dipoles to tune the work function of metals and semiconductors. ${ }^{39}$ In the energy diagram, the dipole introduces a discontinuity of the vacuum level $\Delta \mathrm{E}_{\mathrm{vac}}$ at the interface (Figure $2 \mathrm{~b}$ ) that modifies the band alignment of the junction. In light of the success in passivating the interface achieved by the $\mathrm{I}_{2}$ treatment, we screened other halide and organo-halide compounds such as bromide $\left(\mathrm{Br}_{2}\right)$ and methyl iodide $\left(\mathrm{CH}_{3} \mathrm{I}\right)$. Each of these chemical compounds has been used on c-Si surfaces, resulting in $\mathrm{Si}-\mathrm{Br}$ and $\mathrm{Si}-\mathrm{CH}_{3}$ and $\mathrm{Si}-\mathrm{I}$ bonds, respectively. ${ }^{40,41,42} \mathrm{We}$ analyzed the energetic alignment at the silicon surface for all the three interface treatments using established techniques, particularly XPS and Kelvin probe measurements. ${ }^{43-47}$ These methods (S.I. Figure 8) revealed the presence of a different dipole moments for each of the surface engineering approaches. The results are summarized in Figure 2c. Specifically, a measured $\Delta \mathrm{E}_{\mathrm{vac}}=+0.23 \pm 0.04 \mathrm{eV}$ corresponding to a valence band offset $\Delta \mathrm{VB}=$ $+0.32 \pm 0.04 \mathrm{eV}$ on $\mathrm{I}_{2}$ terminated silicon confirms the presence of the energetic barrier for holes (Figure 3a). Further analysis of the experimental data reveals that the dipole moment formed by adsorbed $\mathrm{Br}_{2}$ and $\mathrm{CH}_{3} \mathrm{I}$ nullifies $\triangle \mathrm{VB}$ at the $\mathrm{c}-\mathrm{Si}$ :CQD interface, thereby simultaneously satisfying requirements (i) and (ii). Efficient collection of photogenerated charges from the CQD film is therefore expected in the case of $\mathrm{CH}_{3} \mathrm{I}$ and $\mathrm{Br}_{2}$ treated silicon diodes.

To verify this prediction, we measured the current-voltage characteristics under AM 1.5 
illumination for diodes with the dipole treatments applied (Figure 2d). All halide treatments show significant short-circuit photocurrent and open circuit voltage $\left(\mathrm{V}_{\mathrm{OC}}\right)$. We note that the relation between the three $\mathrm{V}_{\mathrm{OC}}$ is in agreement with the energetic landscapes previously depicted: the iodine-treated diodes exhibited the highest $\mathrm{V}_{\mathrm{OC}}$ of $0.40 \mathrm{~V}$ whereas the $\mathrm{V}_{\mathrm{OC}}$ of $\mathrm{CH}_{3} \mathrm{I}$ and $\mathrm{Br}_{2}$ diodes was reduced to $0.30 \mathrm{~V}$ and $0.19 \mathrm{~V}$ respectively. This trend is consistent with the direction of the dipole moment formed at the chemically treated c-Si surface and therefore the changes in band alignment between the p-type c-Si and the n-type CQDs.

Carrier lifetime measurements confirmed that all three halide surface treatments significantly reduce trap density compared to $\mathrm{HF}$ treatment. In particular $\mathrm{I}_{2}$ and $\mathrm{CH}_{3} \mathrm{I}$ treatments show a significant enhancement while the $\mathrm{Br}_{2}$ only slightly improves over $\mathrm{HF}$ (S.I. Table 1).
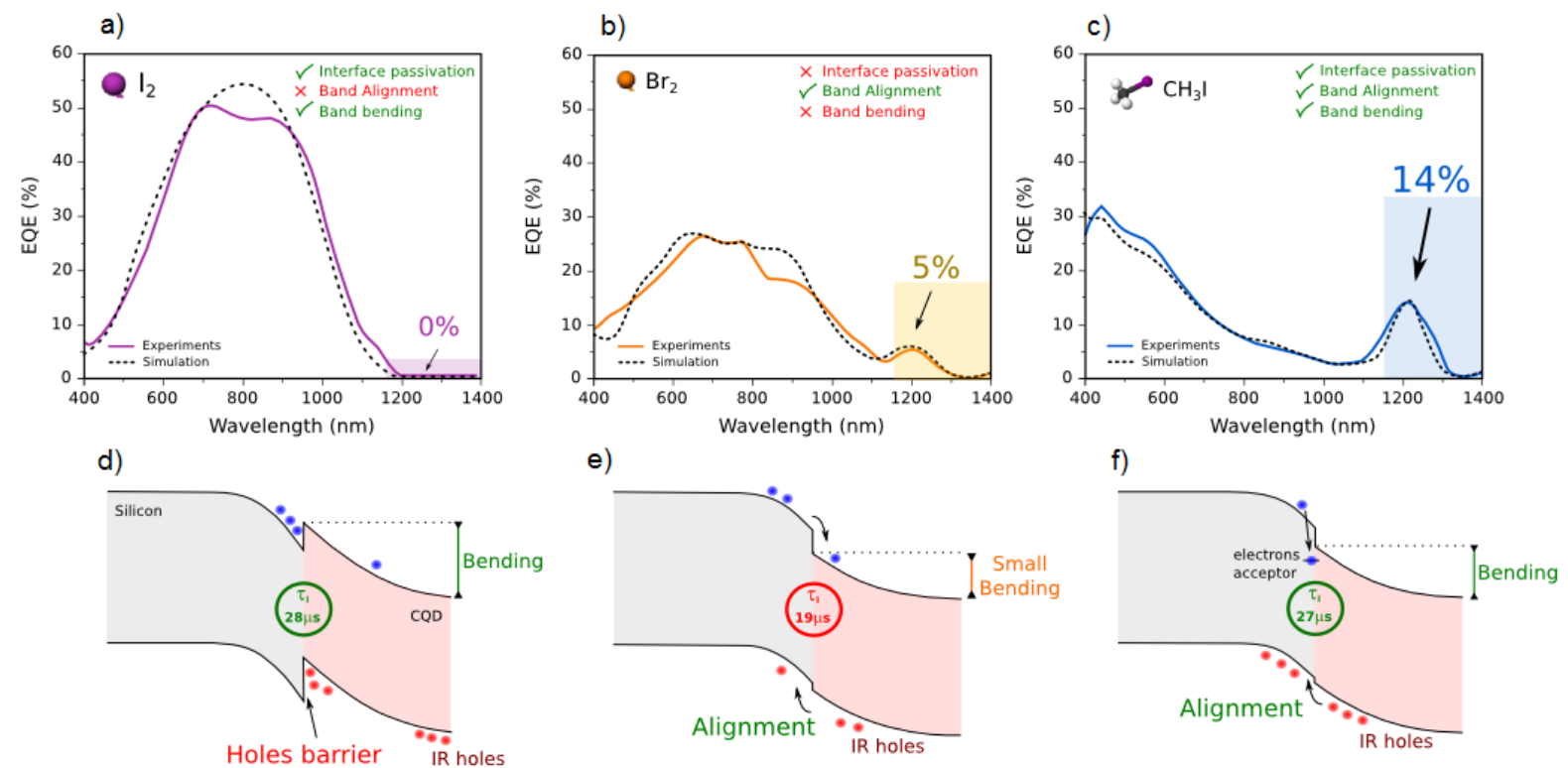

Figure 3. Spectral photoresponse of c-Si:CQD heterojunction diodes. a, b, c) Measured (solid line) and simulated (dashed line) Spectral EQE different halogen treatments: $\mathrm{I}_{2}(\mathrm{a}), \mathrm{Br}_{2}(\mathrm{~b}), \mathrm{CH}_{3} \mathrm{I}$ (c). d), e), f) Energy band diagram at short circuit condition, under $100 \mathrm{~mW} / \mathrm{cm}^{2}$ AM 1.5 solar illumination and interface carrier lifetime (at the center of each diagram) for each diode: $\mathrm{I}_{2}(\mathrm{~d}), \mathrm{Br} 2(\mathrm{e})$, 
$\mathrm{CH}_{3} \mathrm{I}$ (f) . Ideal band alignment is achieved in the case of $\mathrm{CH}_{3} \mathrm{I}$ and $\mathrm{Br}_{2}$ treatment while $\mathrm{I}_{2}$-treated devices exhibit a valence band offset $(0.32 \mathrm{eV})$ blocking holes generated in the CQD layer. The band bending in the CQD layer varies according to band alignment (built-in potential) and is reduced proceeding from the $\mathrm{I}_{2}$ - devices to the $\mathrm{CH}_{3} \mathrm{I}$ - and $\mathrm{Br}_{2}$ - treated ones.

We then investigated the spectral contributions to the photocurrent (EQE) for the three halidetreated c-Si:CQD diodes (Figure 3). These results were further validated using optoelectronic simulations $^{48}$ (dashed line in Figure 3a-c). Beneath each EQE plot, the corresponding energy band diagram, simulated in the short circuit condition, is presented (Figure 3d-f).

The EQE spectrum for the $\mathrm{I}_{2}$ - treated diode shows the collection of charges photogenerated (mostly due to visible light absorption) within the silicon; the bluer wavelengths are largely absorbed in the CQDs, as are the > 1.1 um wavelengths, and as a result these photocarriers are uncollected in light of the valence band barrier from CQDs to silicon.

The spectral response of $\mathrm{Br}_{2}$ - treated diodes exhibits a notable contribution to photocurrent from the CQDs, particularly beyond $1100 \mathrm{~nm}$, where silicon is transparent to photons. As shown in the energy diagram, the interface dipole ensures that no appreciable barrier in the valence band stands in the way of photohole egress from the CQD side into the cSi. Despite this important result, the carrier collection efficiency is reduced at all wavelengths compared to the $\mathrm{I}_{2}$ case previously discussed. We attributed the overall low EQE to the low built-in voltage (see the band alignment for $\mathrm{Br}_{2}$ treatment in Figure 2) that produces only minimal band-bending in the CQD film, limiting photohole transport therein. 
The $\mathrm{CH}_{3} \mathrm{I}$ treated heterojunction shows the highest external quantum efficiency in the SWIR spectral region with $14 \% \mathrm{EQE}$ at $1.23 \mu \mathrm{m}$. This indicates the fulfillment of the three requirements: interface passivation, proper band alignment and a depleted CQD layer. We note a reduced contribution to the spectral response coming from the silicon layer that we can explain if we posit acceptor states at the interface. These states decrease the flux of photoelectrons from the silicon side into the CQD solid. The accumulation of negative charge at the interface is offset by an increased depletion region in the CQD semiconductor, and this enhances extraction of CQDabsorbed photoholes especially apparent at UV and IR wavelengths.
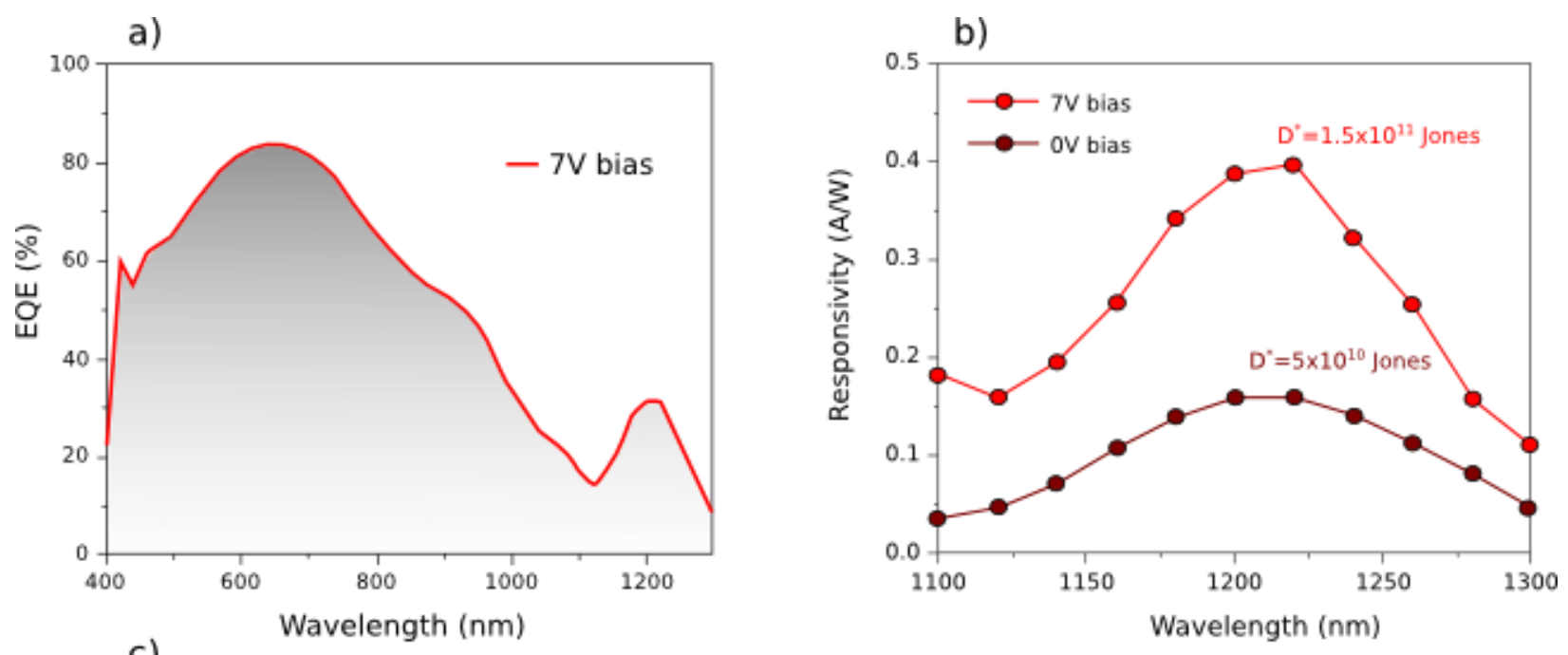

C)

\begin{tabular}{|cccccc|}
\hline Bias & Light intensity & Spectral sensitivity & EQE peak & EQE @ $1230 \mathrm{~nm}$ & D* @1230 nm \\
\hline $0 \mathrm{~V}$ & $100 \mu \mathrm{W} / \mathrm{cm}^{2}$ & $\sim 400-1300 \mathrm{~nm}$ & $31 \% @ 420 \mathrm{~nm}$ & $\sim 14 \%$ & $5 \times 10^{10}$ Jones \\
\hline & & & & & \\
$-7 \mathrm{~V}$ & $100 \mu \mathrm{W} / \mathrm{cm}^{2}$ & $\sim 400-1300 \mathrm{~nm}$ & $84 \% @ 650 \mathrm{~nm}$ & $\sim 33 \%$ & $1.5 \times 10^{11} \mathrm{~J}$ ones \\
\hline
\end{tabular}

Figure 4. Spectral responsivity and detectivity of c-Si:CQDs photodiode a) Spectral external quantum efficiency recorded at 7 $\mathrm{V}$ reverse bias on a $\mathrm{CH}_{3} \mathrm{I}$ treated photodiode. b) Responsivity measured at $0 \mathrm{~V}$ and $7 \mathrm{~V}$ reverse bias under $100 \mu \mathrm{W} / \mathrm{cm}^{2}$ power. The detectivity was calculated at $1.236 \mu \mathrm{m}$, the CQD exciton absorption peak. c) Table summarizing the figures of merit of the detector at different bias conditions. 
Using the best-performing $\mathrm{CH}_{3} \mathrm{I}$ treatment, we fabricated and characterized the first monolithically-integrated c-Si:CQD photodetector. The external quantum efficiency at $1236 \mathrm{~nm}$, under an applied reverse bias of $7 \mathrm{~V}$, reaches $33 \%$, corresponding to a responsivity of $0.4 \mathrm{~A} / \mathrm{W}$ (Figure 4a-b). The specific detectivity of the photodiode at $1236 \mathrm{~nm}\left(D^{*}=\frac{R \sqrt{A}}{\sqrt{2 \cdot q \cdot I_{\text {dark }}}}\right.$ where $A$ is the area of the device $\left(0.049 \mathrm{~cm}^{2}\right), q$ is the electron charge, $R$ is the responsivity and $I_{d a r k}$ is the dark current) is $D^{*}=1.5 \times 10^{11}$ Jones. Under reverse bias, the spectral response presents contributions also from the silicon layer, overcoming $80 \% \mathrm{EQE}$ in the visible range. This responsivity is competitive with that of epitaxial germanium-on-silicon photodetectors. Additionally the device offers a several order-of-magnitude improvement of dark current density. ${ }^{12}$ These attractive performance enhancements come without the need for epitaxy. The fabrication of the detector herein is executed at room temperature and is CMOS compatible. The responsivity of the detector is expected to be a weak function of the incident light power as observedpreviously in $\mathrm{CQD}^{49}$ and Si photodiodes.

In conclusion, the fabrication of a c-Si:CQD heterojunction for short-wavelength infrared sensitization of crystalline silicon allowed us to expand the spectral operating range of silicon based devices. We overcame the limits arising from the natural band alignment of c-Si and CQDs that previously prevented the realization of efficient devices. We did so by using a judicious choice of c-Si surface chemical treatment that also offered the needed CQD type and performance. We further engineered the band alignment and enabled the emergence of a strong built-in electric field. By satisfying three critical operating requirements, we demonstrated the first CQD photodetector monolithically integrated with crystalline silicon to exhibit responsivity beyond silicon's bandgap. 


\section{Methods}

Silicon cleaning and surface treatment. The silicon wafers were cleaned according to the standard RCA cleaning procedure to remove organic and metal contaminants. First the wafer was dip in a solution of ammonium hydroxide : hydrogen peroxide $:$ deionized water at ratio $1: 1: 5$ at $80^{\circ} \mathrm{C}$ for $15 \mathrm{~min}$ (RCA 1). Then it was rinsed in DI water and immersed in oxide stripping solution made of $2 \%$ hydrofluoric acid (HF). Finally the wafer was cleaned in hydrochloric acid : hydrogen peroxide : deionized water at ratio $1: 1: 5$ at $80^{\circ} \mathrm{C}$ for $15 \mathrm{~min}$ (RCA 2).

Before the halogenation the wafer surface was etched with a solution of $2 \% \mathrm{HF}$, rinsed in DI water for 30 to 60 seconds to avoid formation of new oxide and transferred in a nitrogen filled glove box $(\mathrm{O} 2<2 \mathrm{ppm})$ for the subsequent surface treatment. Iodine treatment. The wafer was sealed in a petri dish with a few milligrams of solid iodine and heated up for $20 \mathrm{~min}$. Methyl iodide treatment. The wafer was dipped in a solution of $\mathrm{CH}_{3} \mathrm{I}$ in diethyl ether and exposed to ultraviolet light (254 $\mathrm{nm}$ ) for 20 min. Bromine treatment. The wafer was exposed to bromine vapor at room temperature by sealing it in a petri dish containing a few microliters of bromine in liquid phase.

CQD film deposition and device fabrication. PbS CQDs dispersed in octane were deposited layer by layer on the c-Si wafer by spin casting. The layer was made n-type doped by replacing the original long oleic acid ligands of the CQDs with atomic size inorganic ones coming from tetrabutyl ammonium iodide (TBAI). During the solid state treatment a solution of tetrabutyl 
ammonium iodide in methanol, $10 \mathrm{mg} / \mathrm{ml}$ was dispensed after each layer and spun dry after 30 seconds at $2500 \mathrm{rpm}$ for 10 seconds. Then two rinses with methanol were done at the same speed. The top metal oxide contact was deposited by sputtering at base pressure of $5 \times 10^{7} \mathrm{Torr}$. It consisted of $30 \mathrm{~nm}$ thick of $2 \% \mathrm{AZO}$, which is an ohmic contact with the n-type film and $200 \mathrm{~nm}$ thick ITO layer added to reduce sheet resistance. The back contact was made of $\mathrm{Al}$ and deposited by thermal evaporation on the back, not polished, side of the wafer after RCA cleaning process and oxide stripping. The Al layer thickness was $150 \mathrm{~nm}$. Post metallization annealing was performed at $450^{\circ} \mathrm{C}$ for $15 \mathrm{~min}$ in nitrogen. The device area, $0.049 \mathrm{~cm}^{2}$, was defined on the top layer through a shadow mask.

Absorption measurements were carried out using a Cary 500 UV-Vis - IR spectrometer in an integrating sphere.

I-V and EQE characterization. I-V curves were recorded using a Keithley 2400 digital multimeter. The spectral external quantum efficiency measurements were carried out using a 400W Xenon lamp (Horiba Jobin-Yvon) a monochromator (Horiba JobinYvon FL-1039) optical filters (Newport) and an optical chopper operating at $200 \mathrm{~Hz}$. The chopper was coupled with a Standfod Research Systems lock-in amplifier which was used to measure the photogenerated current. The collimated light was measured through a $0.049 \mathrm{~cm}^{2}$ aperture using a calibrated Newport 818-UV and Newport 818-IR power meters.

Electro-optical model. Device modeling was performed using SCAPS, a one dimensional solar cell simulation program developed at the department of Electronics and Information Systems (ELIS) of the University of Gent, Belgium.

\section{Aknowledgements}


The authors would like to acknowledge the technical assistance and scientific guidance of E.

Palmiano, R. Wolowiec, and D. Kopilovic. This publication is based in part on work supported by Award KUS-11-009-21, made by King Abdullah University of Science and Technology (KAUST), by the Ontario Research Fund Research Excellence Program, and by the Natural Sciences and Engineering Research Council (NSERC) of Canada.

\section{References}

1. Soref, R., Fellow, L. \& Paper, I. The Past, Present, and Future of Silicon Photonics. Sel. Top. Quantum Electron. IEEE J. 12, 1678-1687 (2006).

2. Pavesi, L. \& Lockwood, D. J. Silicon Photonics. 94, (Springer Berlin Heidelberg, 2004).

3. Soref, R. A. Silicon-based optoelectronics. Proc. IEEE 81, 1687-1706 (1993).

4. Masini, G., Colace, L. \& Assanto, G. Si based optoelectronics for communications. Mater. Sci. Eng. B 89, 2-9 (2002).

5. Konstantatos, G. et al. Ultrasensitive solution-cast quantum dot photodetectors. Nature 442, 180-3 (2006).

6. Hansen, M. P. \& Malchow, D. S. Overview of SWIR detectors, cameras, and applications. in SPIE Def. Secur. Symp. (Vavilov, V. P. \& Burleigh, D. D.) 69390I-69390I-11 (International Society for Optics and Photonics, 2008). doi:10.1117/12.777776

7. Lim, Y. T. et al. Selection of Quantum Dot Wavelengths for Biomedical Assays and Imaging. Mol. Imaging 2, 50-64 (2003).

8. Kim, S. et al. Near-infrared fluorescent type II quantum dots for sentinel lymph node mapping. Nat. Biotechnol. 22, 93-7 (2004). 
9. Luryi, S., Kastalsky, A. \& Bean, J. C. New infrared detector on a silicon chip. IEEE Trans. Electron Devices 31, 1135-1139 (1984).

10. Tanabe, K., Watanabe, K. \& Arakawa, Y. III-V/Si hybrid photonic devices by direct fusion bonding. Sci. Rep. 2, 349 (2012).

11. Wang, J. \& Lee, S. Ge-photodetectors for Si-based optoelectronic integration. Sensors 11, 696-718 (2011).

12. Michel, J., Liu, J. \& Kimerling, L. C. High-performance Ge-on-Si photodetectors. Nat. Photonics 4, 527-534 (2010).

13. Wang, X., Cheng, Z., Xu, K., Tsang, H. K. \& Xu, J.-B. High-responsivity graphene/silicon-heterostructure waveguide photodetectors. Nat. Photonics 7, 1-4 (2013).

14. Xia, F., Mueller, T., Lin, Y.-M., Valdes-Garcia, A. \& Avouris, P. Ultrafast graphene photodetector. Nat. Nanotechnol. 4, 839-43 (2009).

15. Nair, R. R. et al. Fine structure constant defines visual transparency of graphene. Science 320, 1308 (2008).

16. Thongrattanasiri, S., Koppens, F. H. L. \& García De Abajo, F. J. Complete optical absorption in periodically patterned graphene. Phys. Rev. Lett. 108, (2012).

17. Streshinsky, M. et al. The Road to Affordable, Large-Scale Silicon Photonics. Opt. Photonics News 24, 32 (2013).

18. Baehr-Jones, T. et al. Myths and rumours of silicon photonics. Nat. Photonics 6, 206-208 (2012).

19. Kim, J. Y., Voznyy, O., Zhitomirsky, D. \& Sargent, E. H. 25th anniversary article: Colloidal quantum dot materials and devices: A quarter-century of advances. Adv. Mater. 25, 4986-5010 (2013).

20. Tang, J. \& Sargent, E. H. Infrared colloidal quantum dots for photovoltaics: Fundamentals and recent progress. Adv. Mater. 23, 12-29 (2011).

21. Sargent, E. H. Colloidal quantum dot solar cells. Nat. Photonics 6, 133-135 (2012).

22. Konstantatos, G. \& Sargent, E. H. Colloidal quantum dot photodetectors. Infrared Phys. Technol. 54, 278-282 (2011).

23. Clifford, J. P. et al. Fast, sensitive and spectrally tuneable colloidal-quantum-dot photodetectors. Nat. Nanotechnol. 4, 40-4 (2009). 
24. Chen, H.-C. et al. Enhanced efficiency for c-Si solar cell with nanopillar array via quantum dots layers. Opt. Express 19, A1141 (2011).

25. Huang, C.-Y. et al. Efficient light harvesting and carrier transport in $\mathrm{PbS}$ quantum dots/silicon nanotips heterojunctions. J. Phys. D. Appl. Phys. 44, 085103 (2011).

26. Song, T. et al. Core-shell structured photovoltaic devices based on $\mathrm{PbS}$ quantum dots and silicon nanopillar arrays. Nanoscale 4, 1336 (2012).

27. Ning, Z. et al. Graded doping for enhanced colloidal quantum dot photovoltaics. Adv. Mater. 25, 1719-23 (2013).

28. Trucks, G., Raghavachari, K., Higashi, G. \& Chabal, Y. Mechanism of HF etching of silicon surfaces: A theoretical understanding of hydrogen passivation. Phys. Rev. Lett. 65, 504-507 (1990).

29. Chang, K. J. \& Chadi, D. J. Theory of hydrogen passivation of shallow-level dopants in crystalline silicon. Phys. Rev. Lett. 60, 1422-1425 (1988).

30. Yablonovitch, E., Allara, D. L., Chang, C. C., Gmitter, T. \& Bright, T. B. Unusually low surface-recombination velocity on silicon and germanium surfaces. Phys. Rev. Lett. 57, 249-252 (1986).

31. Aberle, A. G. Surface passivation of crystalline silicon solar cells: a review. Prog. Photovoltaics Res. Appl. 8, 473-487 (2000).

32. Horányi, T. S., Pavelka, T. \& Tüttö, P. In situ bulk lifetime measurement on silicon with a chemically passivated surface. Appl. Surf. Sci. 63, 306-311 (1993).

33. M'saad, H., Michel, J., Lappe, J. J. \& Kimerling, L. C. Electronic passivation of silicon surfaces by halogens. J. Electron. Mater. 23, 487-491 (1994).

34. Zhitomirsky, D. et al. N-type colloidal-quantum-dot solids for photovoltaics. Adv. Mater. 24, 6181-5 (2012).

35. Ning, Z. et al. All-inorganic colloidal quantum dot photovoltaics employing solutionphase halide passivation. Adv. Mater. 24, 6295-9 (2012).

36. Sinton, R. A., Cuevas, A. \& Stuckings, M. Quasi-steady-state photoconductance, a new method for solar cell material and device characterization. Conf. Rec. Twenty Fifth IEEE Photovolt. Spec. Conf. - 1996 (1996). doi:10.1109/PVSC.1996.564042

37. Anderson, R. L. Experiments on Ge-GaAs heterojunctions. Solid. State. Electron. 5, 341351 (1962). 
38. Tersoff, J. Theory of semiconductor heterojunctions: The role of quantum dipoles. Phys. Rev. B 30, 4874-4877 (1984).

39. Zhou, Y. et al. A Universal Method to Produce Low-Work Function Electrodes for Organic Electronics. Science (80-. ). 336, 327-332 (2012).

40. Colaianni, M. L., Chen, P. J., Gutleben, H. \& Yates, J. T. Vibrational studies of CH3I on Si(100)-(2×1): adsorption and decomposition of the methyl species. Chem. Phys. Lett. 191, 561-568 (1992).

41. Kong, M. J., Lee, S. S., Lyubovitsky, J. \& Bent, S. F. Infrared spectroscopy of methyl groups on silicon. Chem. Phys. Lett. 263, 1-7 (1996).

42. Batra, N. et al. A comparative study of silicon surface passivation using ethanolic iodine and bromine solutions. Sol. Energy Mater. Sol. Cells 100, $43-47$ (2012).

43. Kitatani, T. et al. Analysis of Band Offset in GaNAs/GaAs by X-Ray Photoelectron Spectroscopy. Jpn. J. Appl. Phys. 38, 5003-5006 (1999).

44. Chkoda, L. et al. Work function of ITO substrates and band-offsets at the TPD/ITO interface determined by photoelectron spectroscopy. Synth. Met. 111-112, 315-319 (2000).

45. Mizutani, T., Usunami, T., Kishimoto, S. \& Maezawa, K. Measurement of Contact Potential of GaAs/AlGaAs Heterostructure Using Kelvin Probe Force Microscopy. Jpn. J. Appl. Phys. 38, L767-L769 (1999).

46. Tanimoto, M. Kelvin probe force microscopy for characterization of semiconductor devices and processes. J. Vac. Sci. Technol. B Microelectron. Nanom. Struct. 14, 1547 (1996).

47. Waldrop, J. R. \& Grant, R. W. Measurement of AlN/GaN (0001) heterojunction band offsets by x-ray photoemission spectroscopy. Appl. Phys. Lett. 68, 2879 (1996).

48. Burgelman, M., Nollet, P. \& Degrave, S. Modelling polycrystalline semiconductor solar cells. Thin Solid Films 361-362, 527-532 (2000).

49. Clifford, J. P. et al. Fast, sensitive and spectrally tuneable colloidal-quantum-dot photodetectors. Nat. Nanotechnol. 4, 40-44 (2009). 
